\title{
Reliability and Energy Efficiency of DEAR Protocol with Cooperative Caching in IEEE802.15.4 based large ubiquitous Wireless Sensor Networks
}

\author{
Piyush Charan, Tahsin Usmani, Rajeev Paulus, Syed Hasan Saeed
}

\begin{abstract}
Reliability and Energy Consumption issues in large ubiquitous Wireless Sensor Networks are a cause of concern especially because there is an inherent conflict between the two: an increase in reliability usually leads to an increase in energy consumption. Conversely, energy conservation has been a priority research concern in wireless sensor nodes. Data aggregation from various nodes and its transmission to the sink node through multiple hops which is important for network reliability increases the overall energy consumption in the network. Several schemes were proposed in the past to address the reliability needs and also to minimize the energy consumption in the network. In this context, this paper proposes a novel strategy for IEEE802.15.4/ZigBee based networks by incorporating a Distributed Energy Aware Routing (DEAR) protocol with a localized Cooperative Caching algorithm that addresses the query generated by a requester node or sink node with datum already existing in the locally available cache memory or in the memory of its one-hop neighbors or by the source node. The DEAR protocol considers battery level as a key factor to include nodes in its routing path. The proposed model is evaluated on the basis of three scenarios which were considered to illustrate the impact of energy consumption on the reliability of WSNs.
\end{abstract}

Keywords: Energy Consumption, WSNs, DEAR, Reliability, Cooperative Caching, IEEE802.15.4

\section{INTRODUCTION}

Wireless sensor Networks based on IEEE802.15.4 standard exhibit a low data rate wireless personal area network (LR-WPAN) standard. Energy efficient routing and longevity of a node in such a sensor network is a crucial issue. Two modes in which data is transmitted are 'pull-mode' and 'push-mode'. In push mode, a mote is continuously sensing and sending the data to a coordinator node via multiple hops. Such a node dies off in a network that is continuously transmitting the data sensed to their neighboring nodes or to the sink node, thereby creating a hole

Revised Manuscript Received on December 30, 2019.

* Correspondence Author

Piyush Charan, Department of ECE, Integral University, Lucknow, India. Email: piyush@iul.ac.in

Tahsin Usmani, Department of ECE, Integral University, Lucknow, India.Email: tusmani@iul.ac.in

Rajeev Paulus, Department of ECE, Sam Higginbottom University of Agriculture, Technology and Sciences(SHUATS), Allahabad, India. Email: rajeev.paulus@shiats.edu.in

Syed Hasan Saeed, Department of ECE, Integral University, Lucknow, India. Email: s.saeed@iul.ac.in

(C) The Authors. Published by Blue Eyes Intelligence Engineering and Sciences Publication (BEIESP). This is an open access article under the CC BY-NC-ND license (http://creativecommons.org/licenses/by-nc-nd/4.0/) in the network known as the 'energy hole'. The problem of energy hole is minimal in the case of pull mode where the sensor mote is active only for the period of transmission and goes back to sleep mode if there is no data to be sensed or transmitted. IEEE802.15.4/ZigBee standard offers a beacon-enabled mode in which the sensor device or mote turns active on the arrival of a beacon signal and participates in sensing or transmission of data depending on the case.

For large ubiquitous network, apart from energy efficiency the metric for performance estimation is Reliability. Reliability primarily refers to the transmission of data in the form of bundles from one end of the wireless sensors that are from the Sensors (or source node) to the other end of the network that is to the PAN Coordinator (or sink node). Sensor network based on the IEEE802.15.4 based sensors is formed by various one-hop neighbors. For traditional packet reliability, many researchers ensured that all packets carrying sensed data from sensor nodes need to be reliably transmitted to the receiver. Packet loss can occur due to the error-prone wireless links, so end-to-end packet reliability is required that enables acknowledgement of each data packet and each lost packet is retransmitted.

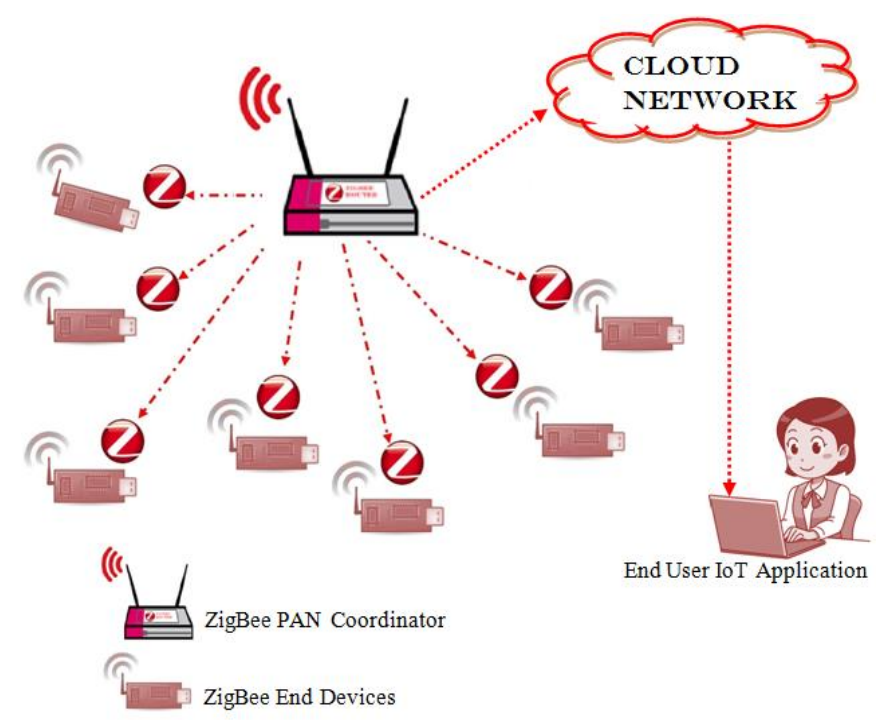

Fig.1. IEEE802.15.4/ZigBee based network Architecture

Achieving reliability with cooperative caching is challenging because sometimes the data requested by the sink node in the pull mode is procured from the nearby caching node but that data is not reliable due to the fact that its time-to-live $(t t l)$ value may have expired which means that the data supplied by the caching node to the sink may have expired and fresh data from other cache nodes or from the source node is to be supplied.

Published By:

Blue Eyes Intelligence Engineering

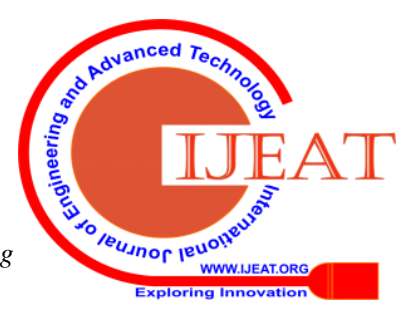


Thus, reliability is an important issue that needs to be addressed as it also provides a trade-off between efficient energy consumption and the successful delivery of the datum requested by the requester node or the sink node. In this paper, we consider three scenarios in which we consider two analytical models for the simulation of nodes and one random model. The two analytical models considered are the grid model (or peer-to-peer) and the star model (or cluster). In the random model the motes are deployed randomly. The number of motes is varied from 100 nodes to 200 nodes and the simulation is modeled with Distributed Energy Aware Routing (DEAR) protocol with CCZ cooperative caching as data retrieval model.

\section{RELATED WORKS}

The Quality of Service (QoS) for large ubiquitous networks based on the IEEE 802.15.4 can be achieved by optimizing the energy consumption (via enhancing the network lifetime) and increasing the reliability (by escalating the chance of a packet being delivered). To achieve reliability, two solutions may be proposed in the protocol stack. First, some nodes of the entire network are active and must be involved in the routing of data while other motes are still in sleep mode. In this technique some parts of the networks may not be accessible because the WSN node may be sleeping making it the most energy efficient strategy but less reliable [1]. The second strategy is to create multiple paths (through a routing algorithm) in a multi-hop environment between a particular sensor node and the sink node (or the PAN Coordinator in our case). The latter solution is most reliable but more power consuming than the former as it will produce more number of packet overheads. To curb the creation of excessive packet overheads due to retransmissions in a sensor environment a cooperative caching strategy is employed in the network wherein, the data requested by the PAN coordinator or the sink node is retrieved from the on bard cache memory via a local hit, zone hit, remote hit or a global hit [2]. Caching in Cooperative zones (CCZ) is a caching scheme which allows nodes to store frequently accessed data items in the cache memory available at the on-board sensor module. Daniel Messina et al. in [3] proposed Power Efficient Routing and Limited Latency (PERLA) network layer protocol that was stacked to the IEEE802.15.4 protocol stack which aggregated data by employing caching and retransmission technique. Their design addressed the synchronization of motes, power management and link layer reliability. Nikos Dimokas et al. in [4] described a high performance and less complicated cooperative caching strategy for WSNs that serves data with minimal latency and reduced energy consumption for several applications such as controlling smart buildings and target tracking in battlefields. Their design proves to be $20 \%$ more reliable than others for IEEE 802.11. Later, in [5] Narottam Chand presented an energy efficient cluster cooperative caching at sensor (C3S) based upon grid type clustering and demonstrated that the motes of the same cluster or grid forms a cooperative cache system. Their proposal showed improved energy efficiency over $\mathrm{NiCoCa}$ routing protocol and also demonstrated an enhanced reliability based on the Byte Hit Ratio (BHR) of the caching node. In this paper, the byte hit ratio is taken as a performance metric to evaluate the reliability of the system for the three proposed scenarios, as discussed above. S. Gowrishankar et al., in [6] evaluated the performance of various on-demand routing protocols in a network based on IEEE802.15.4 PHY and MAC standards and they established a comparison on the Packet Delivery Ratio, Average Network Delay and Throughput of the network based on their simulations on NS2. They estimated the reliability in terms of the Packet Delivery Ratio (PDR) as they have not employed caching as a technique to retrieve data. Their model has both push and pull environment which means that data is continuously sensed and pushed to the coordinator node to take control actions. The simulation was run for various traffic loads in the range $0.001 \mathrm{pps}$ to $5 \mathrm{pps}$ and showed that when the data rate is 1pps then AODV nearly shows a PDR of nearly $50 \sim 60 \%$. This means that out of the sensed data only $50 \sim 60 \%$ data is received at the Sink node or the PAN coordinator and rest data packets are lost. The proposed DEAR protocol with cooperative caching scheme has fairly overcome this problem and it has satisfactorily decreased the packet loss.

\section{ROLE OF RELIABILITY IN WSN}

Reliability in a network of nodes arranged in a grid (peer-to-peer), star (cluster) or for nodes deployed in a random topology based on the IEEE802.15.4/ZigBee standard are estimated based on the successful deliveries of the data packets requested by the Sink Node or the PAN coordinator. Reliability is represented as a ratio of the total number of data packets received by the destination node to the number of data packets sent by the source node [7]. In our scenarios, the reliability is estimated as the ratio of the number of data packets received by the PAN coordinator to the total number of data packets requested by the Sink Node or the PAN coordinator or to the number of data items sent by a nearby caching node.

$$
\text { Reliability }=\frac{\text { no. of data packets received }}{\text { no. of data packets requested }}
$$

\section{ROLE OF CACHING IN ESTIMATING RELIABILITY}

Previously, in a sensor network when the data packets were to loss then the method of retransmission was to be carried out creating more number of redundant packets flooded in the entire network. This method was most reliable as the packet lost would somehow be retransmitted and made to reach the destination. But this required more energy consumption by the nodes and a lot of bandwidth was wasted. This problem was minimized by incorporating the caching strategy. Caching facilitates storing of data items that are frequently accessed in an on-board cache provided in the sensor mote [8]. In WSNs based on IEEE802.15.4 an adaptive technique for caching in Cooperative Zone (CCZ) is incorporated for data retrieval. 
The data requested by the PAN coordinator or the sink node is pre-fetched in the locally available cache memory of the node itself. If the required data is found in the local cache,

then such fetching of data is termed as a Local Hit. When the data required is not found via the local hit then the data is tried to be retrieved from the one hop neighbors present in the zone of the requesting node. Such retrieval is known as a Zonal Hit. If somehow zonal hit also fails, then this caching strategy looks for possible retrieval of data from the node present in a region other than the local zone of the requester along the route path towards the source of the data, this is known to have encountered a Remote Hit. Apparently, if the remote hit also fails then an attempt to retrieve the data from the cache of the source node is made and this type of retrieval is known as a Global Hit. It is always favorable for a sensor node (SN) under this scheme to extend cached data to its one hop neighbor available in its zone. The sensor mote which is a member of a particular zone of a mote forms a collaborative cache system for that particular node because link costs or communication costs with them are very low in terms of consumption of energy and the exchange of data.

\section{DEAR PROTOCOL WITH COOPERATIVE CACHING}

The DEAR (distributed energy aware routing) protocol is a modified version of the traditional AODV (ad-hoc on demand distance vector) routing protocol wherein the routing path is chosen based on the residual energy of the nodes [9]. A node is included in the path of such a routing protocol if the residual energy of the node is at least $80 \%$ or more of the total link cost to send the requested datum from source to destination. The total energy cost attribute, $\mathrm{TEC}_{i}$ in the DEAR protocol needs to be optimized so as to minimize the overall cost of power consumption while satisfying the requested Quality of Service (QoS) which will integrate the power requirements and the QoS constraint [10]. The total energy cost, $\mathrm{TEC}_{i}$ may be expressed as:

$$
\operatorname{TEC}_{i}=P_{\lambda(i)}^{m}\left(t_{m}, \overline{T_{\lambda(i)}}\right)+\gamma Q_{\lambda(i)}^{m}\left(t_{m}, \overline{T_{\lambda(i)}}\right)
$$

Where, $\gamma>0$ is a constant that matches the delay units with respect to power.

The Threshold must be used as a condition to accept a node in a probable routing path so as to achieve Distributed Energy Aware Routing. The threshold may be repesented as:

$$
\text { Threshold }=0.8 \times T E C_{i j}
$$

The Cooperative Cache Zone (CCZ) localized algorithm supplements the validity of the network model and facilitates the retrieval of the requested data from neighboring nodes in each zone. The algorithm for DEAR protocol to select the nodes in the routing path is given below.

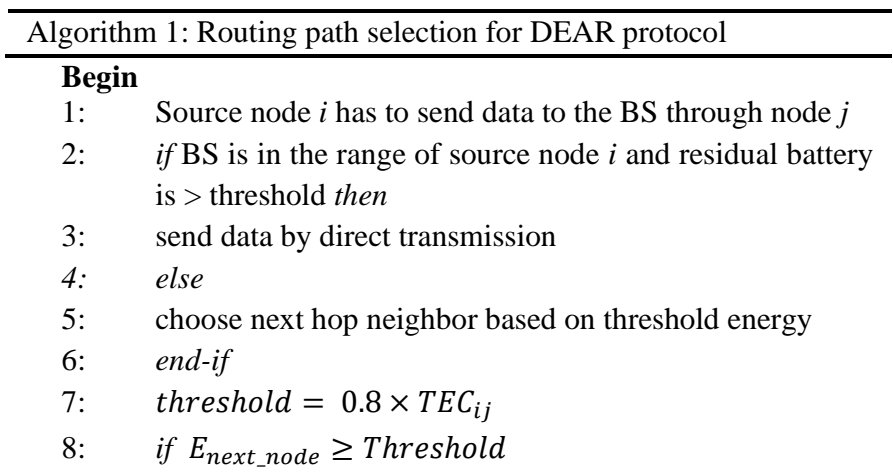

9: $\quad$ next_node, $i^{*}$ gets RREQ packet and sends ACK to source node, $i$

10: end-if

11: Node $i^{*}$ determines its next hop node with maximum residual energy iteratively until destination_node, $j$ gets RREQ

12: RREQ message reaches the PAN Coordinator (or BS)

13: destination_node, $j$ replies with RREP to source_node, $i$ and transmission begins

End

The base station or the PAN coordinator in our case is represented as BS, here. Using the DEAR protocol has substantially increased the network lifetime.

\section{SIMULATION SETUP AND RESULTS}

This section presents the simulation parameter and the network scenarios on which the simulation is carried out in NS2.

\section{A. Simulation Setup}

We have considered three scenarios all together wherein the number of nodes is varied from 100 to 200. The wireless motes are based on the IEEE802.15.4 PHY and MAC standards.

Scenario 1: In this Scenario the nodes are arranged in a star or cluster based model where a node in the center acts as a hub and is communicating with neighboring nodes as shown in figure 2(a).

Scenario 2: In this scenario the nodes are arranged in peer-to-peer or grid based network model wherein the nodes based on IEEE 802.15.4 MAC and PHY standards are aligned in a grid where node-to-node distance is $1 \mathrm{~m}$, as shown in figure 2(b).

Scenario 3: The third scenario involves the wireless sensor nodes to be arranged in a random manner as shown in figure 2(c). These IEEE802.15.4 based wireless nodes are randomly deployed in an area of $100 \mathrm{~m} * 100 \mathrm{~m}$.

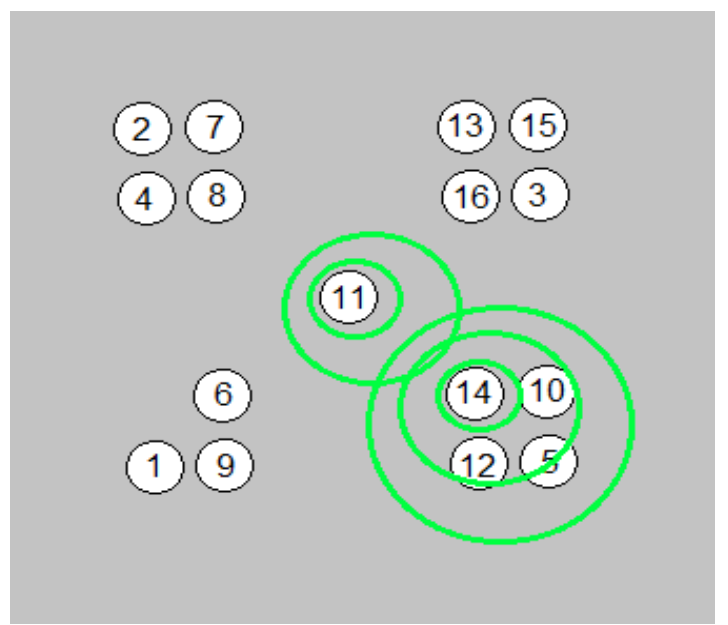

(a) Star or Cluster based Model 


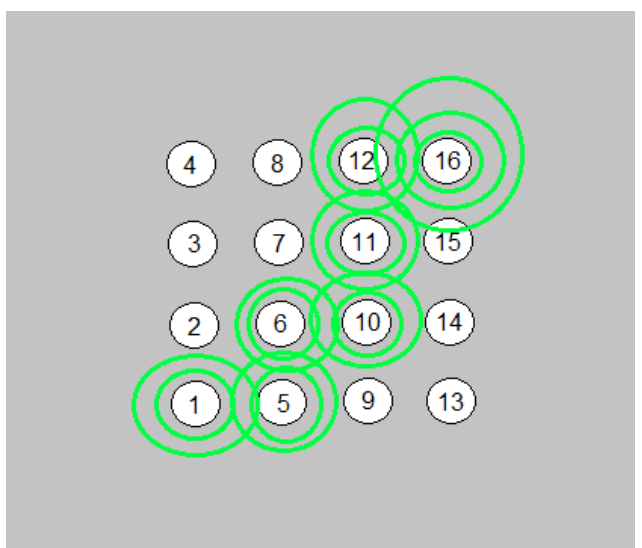

(b) Grid or Peer-to-Peer based Model

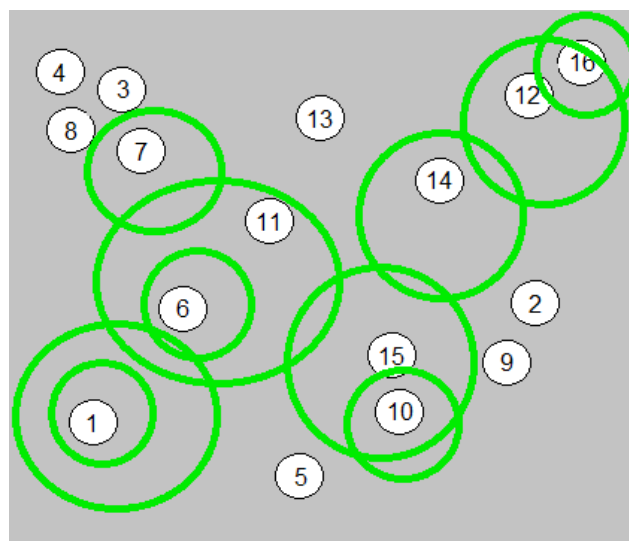

(c) Randomly Deployed Nodes

\section{Fig.2. Scenarios of arrangement of Nodes}

In all the three scenarios the number of nodes is increased from 100 200 and the simulation time is kept constant to 510s. In all the networks scenarios a PAN Coordinator which is a full functional device (ffd) is incorporated for taking control decision by performing a series of processing on the aggregated data. A constant bit rate (CBR) traffic type is used and the time-to-live (ttl) for the cached data is 300s.The beacon order (BO) and the superframe order (SO) is kept constant as 3 and 2 respectively so that the duty cycle offered is $50 \%$.

Duty cycle $=2^{-(\mathrm{BO}-\mathrm{SO})}=2^{-(3-2)}=2^{-1}=\frac{1}{2}=0.5$ or $50 \%$

The routing protocol used is DEAR and is assisted with a localized caching algorithm known as the Caching in Cooperative Zones (CCZ). The simulation parameters are summarized below:

\section{Table 1. Simulation Parameters}

\begin{tabular}{|c|c|c|}
\hline Parameter & Default Value & Range \\
\hline Number of Nodes & 100 & $100 \sim 200$ \\
\hline Area & $100 * 100 \mathrm{~m}^{2}$ & \\
\hline Number of Data Items & 500 & \\
\hline Payload Size & 64bytes & \\
\hline PHY and MAC Layer & IEEE802.15.4 & \\
\hline Channel Frequency & $2.4 \mathrm{GHz}$ & \\
\hline Bandwidth (kbps) & 250 & \\
\hline Simulation Time $\left(t_{S}\right)$ & $500 \mathrm{~s}$ & \\
\hline Waiting interval $\left(t_{w}\right)$ & $10 \mathrm{~s}$ & \\
\hline Total Time $\left(t_{s}+t_{w}\right)$ & $510 \mathrm{~s}$ & \\
\hline TTL & $300 \mathrm{~s}$ & \\
\hline Cache Size (KB) & 800 & \\
\hline Traffic Type & CBR & \\
\hline Data Rate & $1 \mathrm{pps}$ & \\
\hline Routing Protocol & DEAR & \\
\hline Beacon Order & 3 & \\
\hline
\end{tabular}

\section{Superframe Order}

\section{B. Performance Metrics}

1. Byte Hit Ratio (B): The quality of service (QoS) of the proposed algorithm is measured in terms of its reliability which is defined as the ratio of the no. of data packets received by the sink node or the PAN coordinator to the number of packets requested by the requester. But since we have incorporated caching as a technique for possible data retrieval therefore the reliability of the network with DEAR routing protocol is measured in terms of the Byte Hit Ratio which is described as the ratio of total data bytes retrieved from the cache to the total number of requested data bytes by the PAN Coordinator node. The byte hit ratio (B) is the algebraic sum of total bytes retrieved from a local hit known as a Local Byte Hit ( $\left.B_{\text {local }}\right)$, bytes retrieved from zonal hit known as a Zone Byte Hit ( $\left.B_{\text {zone }}\right)$ and the bytes retrieved from a remote hit known as a Remote Byte Hit $\left(\mathrm{B}_{\text {remote }}\right)$ and may be expressed as:

$$
B=B_{\text {local }}+B_{\text {zone }}+B_{\text {remote }}
$$

The more the byte hit ratio for a particular scenario, the more is the reliability exhibited by the network for the DEAR routing protocol with Cooperative Caching.

2. Energy Consumption (E): the total Energy consumption by a wireless senor mote based on IEEE802.15.4 PHY and MAC standards is the energy consumed for transmitting, receiving and aggregating the data. Network lifetime is analogous to Energy Consumption by the nodes in a way that the sensor nodes that are actively participating in the network communication are losing the energy stored in the onboard batteries to an extent that they eventually die-out and creates a node hole.

$$
E_{\text {Residual }_{i}}=E_{\text {initial }}-E_{i}
$$

Where, $E_{\text {Residual }_{i}}$ is the residual energy available for utilization in node ' $i$ '. $E_{\text {initial }}$ is the initial energy of node ' $i$ ' and $E_{i}$ is the energy consumption by node ' $i$ ' in transmitting, receiving and retrieving data from the cache node.

$$
E_{\text {Tot }}=\sum_{i=0}^{n} E_{i}
$$

Where, $E_{\text {Tot }}$ is the Total energy Consumption by all the ' $n$ ' nodes present in the network. The more the Energy Consumption for a particular network, the lesser is the Network lifetime of the nodes present in that scenario.

\section{Simulation Results}

The IEEE802.15.4 based network is simulated on NS2 for the various scenarios considered and the following results have been obtained for Reliability and Energy Consumption. 
Reliability is analogous to the Byte Hit Ratio in a way that the system exhibits maximum reliability if the data bytes requested are retrieved completely. The reliability can be estimated by analyzing the Byte Hit Ratio (B) for the data retrieved from the cache in cluster, peer-to-peer and random topologies. The simulation has been run for each of the three scenarios and the number of nodes in the network has been varied from 100 to 200 each time.

Table 2. Reliability in terms of Byte Hit Ratio (B) in each scenario based on the number of nodes with DEAR protocol and the $\mathrm{CCZ}$ caching algorithm.

\begin{tabular}{cccc}
\hline $\begin{array}{c}\text { No. of } \\
\text { Nodes }\end{array}$ & \multicolumn{2}{c}{ Byte Hit Ratio (in \%age) } \\
\hline & $\begin{array}{c}\text { Scenario 1- } \\
\text { Randomly Deployed } \\
\text { Network }\end{array}$ & $\begin{array}{c}\text { Scenario 2- } \\
\text { Star Based } \\
\text { Model }\end{array}$ & $\begin{array}{c}\text { Scenario 3- } \\
\text { Grid Based } \\
\text { Model }\end{array}$ \\
\hline $\mathbf{1 0 0}$ & 100 & 100 & 100 \\
$\mathbf{1 2 0}$ & 100 & 100 & 100 \\
$\mathbf{1 4 0}$ & 100 & 99.2126 & 98.7261 \\
$\mathbf{1 6 0}$ & 98.7842 & 96.1877 & 98.3003 \\
$\mathbf{2 0 0}$ & 99.6497 & 100 & 99.0923 \\
\hline
\end{tabular}

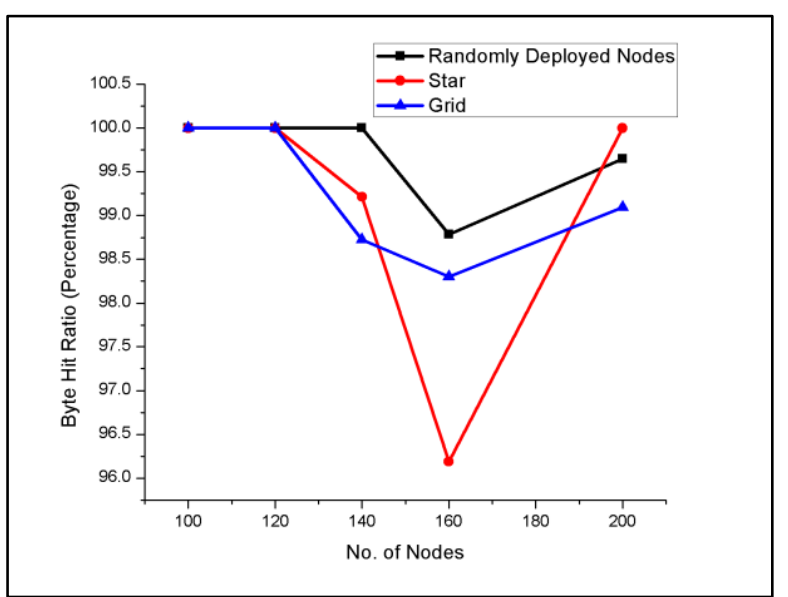

Fig.3. Graph showing the Reliability in terms of Byte Hit Ratio exhibited in different Network Scenarios for DEAR protocol with Caching v/s the number of nodes

The graph in figure 3 shows the Reliability in terms of the Byte Hit Ratio (B) exhibited in different network scenarios for DEAR protocol when executed with the localized caching algorithm. The simulation results clearly show that when the number of nodes is increased in the network then for the Star Based network the Byte hit ratio (BHR) drops to nearly 96\% at 160 nodes which is still a good percentage but when we compare this with other network topologies then star based or cluster based network loses its sheen. The randomly deployed nodes show better and consistent reliability in terms of the byte hit ratio even when the number of nodes is increased. The drop in the percentage of BHR in all the three scenarios shows that when the number of nodes is increased the packet drops increases slightly.

However, if we compare these results with the results of S. Gowrishankar et al., [6] in which they estimated the reliability in terms of the packet delivery ratio (PDR) there is an increase of nearly $20 \%$ in the reliability exhibited by the proposed system with cooperative caching, wherein the pull mode scenario of the nodes exploits the cache memory available on the on-board sensor module for retrieving the data required to be fetched by the PAN coordinator.
The caching of data involves temporarily storing it in the onboard cache memory of the node for the ' $t \mathrm{tl}$ ' (time-to-live) period of the cache data which also requires power consumption. The typical $t t l$ value is 300 s in our simulation setup and therefore after the ' $t t l$ ' expires new cache data is to be updated as per the caching scheme. The overall energy expenditure accounts to transmission, reception, data aggregation, caching and retrieving the requested bytes from the cache by the nodes in the network. The assumption of $50 \%$ duty cycle is made so that the node becomes active for half the time and goes to standby mode for the remaining time when a beacon signal is sent across the node which is present in the routing path from source to destination (PAN Coordinator). The total Energy Consumption of the network is computed as shown in table below for the nodes arranged in various analytical models and also in random topology.

Table 3. Energy Consumption for different Network Scenarios

\begin{tabular}{|c|c|c|c|}
\hline $\begin{array}{l}\text { No. of } \\
\text { Nodes }\end{array}$ & \multicolumn{3}{|c|}{ Energy Consumption (mW) } \\
\hline & $\begin{array}{l}\text { Scenario 1- Randomly } \\
\text { Deployed Network }\end{array}$ & $\begin{array}{c}\text { Scenario 2- } \\
\text { Star Based } \\
\text { Model }\end{array}$ & $\begin{array}{c}\text { Scenario 3- } \\
\text { Grid Based } \\
\text { Model }\end{array}$ \\
\hline 100 & 4.0205 & 4.1657 & 4.0045 \\
\hline 120 & 4.1342 & 4.1789 & 4.0879 \\
\hline 140 & 5.4444 & 4.4759 & 4.4629 \\
\hline 160 & 4.661 & 4.7451 & 4.6164 \\
\hline 200 & 5.2827 & 5.0673 & 4.9405 \\
\hline
\end{tabular}

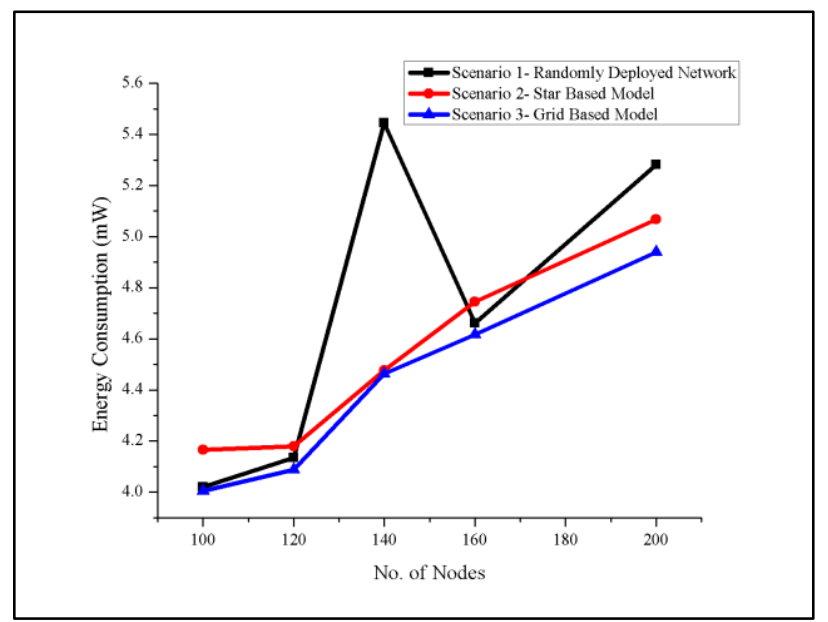

Fig.4. Graph showing comparison of Energy Consumption in different Network Scenarios v/s the number of nodes

The graph in figure 4 typically shows the energy consumption by the nodes in a network arranged in different analytical models and also for the network wherein the nodes are randomly deployed as shown in fig. 2(c). The graph clearly shows that the energy consumption in case of the randomly deployed nodes is higher than the other two analytical models. The average energy consumption for randomly deployed nodes is $4.7086 \mathrm{~mW}$.

Published By:

Blue Eyes Intelligence Engineering \& Sciences Publication 


\section{Reliability and Energy Efficiency of DEAR Protocol with Cooperative Caching in IEEE802.15.4 based large ubiquitous Wireless Sensor Networks}

The star or cluster based network model exhibits a average energy consumption of $4.5266 \mathrm{~mW}$. Whereas, the peer-to-peer or grid based network exhibits an average energy consumption of $4.4224 \mathrm{~mW}$.This shows that a grid based network exhibits lesser energy consumption.

\section{CONCLUSION}

Although the energy consumption is less in case of the grid based network model which is double of what Charan, P. et al. in [11], have obtained. They showed an average energy consumption of $1.9214 \mathrm{~mW}$ for 16 nodes with AODV routing protocol. This increase in energy consumption is in proportion to the increase in the number of nodes. For a particular scenario the byte hit ratio determines the Reliability which is better in case of randomly deployed nodes and the total energy consumption is less in case of the Grid based network model. This means that the network lifetime for the nodes participating in a peer-to-peer network is slightly higher than the other analytical models. thus to achieve higher reliability we consider the network in which the wireless motes are randomly arranged whereas, for achieving higher energy efficiency we consider the peer-to peer or grid based network model.

\section{ACKNOWLEDGMENT}

This work has been partially supported by the Integral Information and Research Center (IIRC) of Integral University vides the Manuscript Communication No. IU/R\&D/2019-MCN000771.

\section{REFERENCES}

1. Trivedi K.S. Probability and Statistics with Reliability, Queueing Computer Science Applications. Wiley; Hoboken, NJ, USA: 2002. p. 830.

2. Charan, P., Usmani, T., Paulus, R., and Saeed, S.H., "A Cooperative Cache Management Scheme for IEEE 802.15.4 based Wireless Sensor Network", International Journal of Electrical and Computer Engineering, Vol. 8 Issue 3, pp.1701-1710, 2018. doi: 10.11591/ijece.v8i3.pp1701-1710.

3. Messina, D., Ortolani, M., and Re, G. L., "Achieving Robustness through Caching and Retransmissions in IEEE 802.15.4-based WSNs", 16th International Conference on Computer Communications and Networks, pp. 1117-1122, 2007. doi: 10.1109/ICCCN.2007.4317968.

4. Dimokas, N., Katsaros, D., Tassiulas, L., \& Manolopoulos, Y., "High Performance, Low Complexity Cooperative Caching for Wireless Sensor Networks", IEEE International Symposium on a World of Wireless, Mobile and Multimedia Networks \& Workshops, 1-9, 2009.

5. Chand, N. (2013). Energy Efficient Cooperative Caching in WSN. International Journal of Computer, Electrical, Automation, Control and Information Engineering, WASET. 7. 665-670.

6. S. Gowrishankar., S. K. Sarkar and T. G. Basavaraju, "Performance analysis of AODV, AODVUU, AOMDV and RAODV over IEEE 802.15.4 in wireless sensor networks," 2009 2nd IEEE International Conference on Computer Science and Information Technology, Beijing, 2009, pp. 59-63. doi: 10.1109/ICCSIT.2009.5234469

7. Wategaonkar, D. N. and Nandhini, R., "A Survey on Reliability in Wireless Sensor Network", Indian Journal of Science and Technology, Vol 9, Issue 37, pp. 1-6,2016. doi:10.17485/ijst/2016/v9i37/98926

8. Charan, P., Usmani, T., Paulus, R., and Saeed, S.H., "Cooperative Caching in IEEE802.15.4 based WSNs", International Journal of Applied Engineering Research, Vol. 12 Issue 21, pp.11409-11416, 2017.

9. Charan, P., Usmani, T., Paulus, R., and Saeed, S.H., "Empirical Validation for Energy Efficiency of DEAR routing protocol over
AODV in IEEE802.15.4 based Wireless Sensor Networks", International Journal of Innovative Technology and Exploring Engineering, Vol. 8 Issue 8, pp.853-857, 2019.

10. Charan, P., Usmani, T., Paulus, R. and Saeed, S.H., "Performance Evaluation of AODV Protocol for Energy Consumption and QoS in IEEE 802.15.4 Based Wireless Sensor Network Using QualNet Simulator" Wireless Sensor Network, Vol.8 Issue 8, 166-175, 2016. doi: 10.4236/wsn.2016.88014.

11. Charan, P., Usmani, T., Paulus, R., and Saeed, S.H., "Performance of Distributed Energy Aware Routing (DEAR) Protocol with Cooperative Caching for Wireless Sensor Networks", Wireless Sensor Network, Vol.11 Issue 3, 35-45, 2019. doi: 10.4236/wsn.2019.113003.

12. Fatima, L.N., Mahin, S. H., Taranum, F., Khan, K.U.R., "Power Management Strategies in MANETs", International Journal of Recent Technology and Engineering, Vol.8 Issue 1S4, pp. 703-708, 2019.

\section{AUTHORS PROFILE}

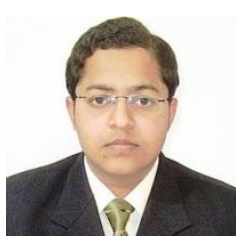

Piyush Charan works as Assistant Professor, Department of ECE, Integral University of Lucknow. He is a member of IEEE, member of ACM and life member of ISTE. He is currently pursuing a doctorate degree in engineering. His research interest are Wireless Sensor Networks, LoWPAN devices and integration of IoT systems.

E-mail:piyush@iul.ac.in

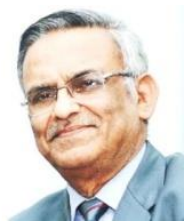

Tahsin Usmani, currently works as Professor in the Department of ECE of Integral University. In January 2011, he was superannuated as Executive Director from ITI, Ltd. (Govt. of India Corporate Sector) in January, 2011. In 2012, he was awarded a doctorate degree from Allahabad University in Electronics and Communication Engineering. His research interest includes Energy Conservation and Effective utilization of Solar Energy, Ubiquitous Wireless Sensor Networks and Mobile Computing E-mail: tusmani@iul.ac.in

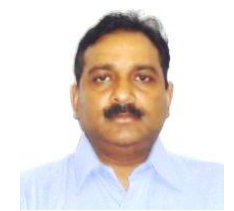

Rajeev Paulus, works as Assistant Professor in the Department of ECE of SHUATS, Allahabad. He is a Post Graduate in Engineering from MNNIT, Allahabad and was awarded a doctorate degree in Electronics \& Communication Engineering from the Department of Electronics Engineering of Sam Higginbottom University of Agriculture, Technology and Sciences, Allahabad, India. His research interest includes Wireless Sensor Networks and Cognitive Computing Networks.

E-mail: rajeev.paulus@shiats.edu.in

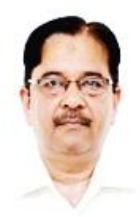

Syed Hasan Saeed, works as Professor \& HoD, Department of ECE, Faculty of Engineering at Integral University. In 2010, he was awarded a PhD in Electronics Engineering from Integral University. He is the author of many books on Control Systems and Control Engineering. His research interest includes Energy Optimization, E-Nose, Sensors and Transducers, Control Systems, Wireless Sensors and Efficient Routing in Mobile Ad-Hoc Networks. E-mail: ssaeed@iul.ac.in 